\title{
A NEW APPROACH TO NUMERICAL INTEGRATION BASED ON COIFMAN WAVELETS
}

\author{
Bachir Dehda \\ Department of Mathematics, Faculty of Exact Sciences, University of Echahid Hamma Lakhdar \\ El-Oued, Algeria \\ dehda-bachir@univ-eloued.dz
}

Received: 29 November 2018; Accepted: 18 July 2019

\begin{abstract}
In this paper, we present a new approach based on Coifman wavelets to find approximate values of definite integrals. This approach overcomes both CAS and Haar wavelets and hybrid functions in terms of absolute errors. The algorithm based on Coifman wavelets can be easily extended to find numerical approximations for double and triple integrals. Illustrative examples implemented using Matlab show the efficiency and effectiveness of this new method.
\end{abstract}

MSC 2010: 65D30, 65D32

Keywords: numerical integration, CAS wavelets, Haar wavelets, hybrid functions, Coiflets

\section{Introduction}

Numerical integrations play an important role in various areas of applied sciences and engineering. Because most integrals cannot be determined via analytical methods, the numerical integration methods have taken a growing interest of many researchers for approximating the value of a definite integral. To see some quadrature rules based on polynomials, one can refer to [1-3]. In recent years, wavelets have gained a lot of popularity and have become a standard tool for many disciplines. So, many authors applied wavelets in images processing [4,5], in mathematics [6] and in other areas of physics and engineering. Wavelet bases with collocation methods have been used for solving single, double and triple integrals [7-9], such as in [10] and for $2^{k}(2 M+1)$ points, the error bound corresponding to CAS wavelets is inversely proportional to $M$. Similarly, in [8] for $2 M$ points, the Haar wavelets method provides an approximation error evaluated by $O\left(M^{-1}\right)$. In addition, the Hybrid function method uses $N M$ points with $k$-th derivative of the integrand function to get a better error bound estimated by $O(N M)^{-k}$. However, the convergence rate of 
previous methods is only $O\left(M^{-s}\right)$ at most and it does not decrease exponentially to obtain a faster approximation. Moreover, it cannot be improved by increasing the order derivative of the integrand function. To overcome this problem and to improve the convergence rate, we propose in this paper a new method based on Coifman wavelets for solving single, double and triple integrals. By using this new method, the convergence rate is improved to $O\left(2^{-2 N j}\right)$ for an integrand function $f \in C^{2 N}$ and $j \geq 0$. Illustrative examples have shown the accuracy and effectiveness of our proposed method compared to existing methods.

This work is structured as follows: The next section recalls the CAS and Haar wavelets methods to numerical integration. Section 3 introduces the hybrid functions method. Section 4 presents the proposed method for single, double and triple integrals with error analysis. Section 5 provides numerical examples to investigate the efficiency of our proposed method. Finally, we bring this work to a close with a conclusion and main references.

\section{Numerical integration using CAS and Haar wavelets}

In this section, we recall the CAS and Haar wavelets methods to numerical integration for single, double and triple integrals.

\subsection{CAS wavelets}

In [7], Cosine and Sine wavelet $\psi_{k, n}(x)$ is defined by:

$$
\psi_{k, n}(x)=\left\{\begin{array}{cl}
2^{\frac{k}{2}} C A S_{m}\left(2^{k} x-n\right), & \frac{n}{2^{k}} \leq x<\frac{n+1}{2^{k}} \\
0, & \text { otherwise, }
\end{array}\right.
$$

where: $C A S_{m}(x)=\cos (2 m \pi x)+\sin (2 m \pi x), k=0,1,2,3, \ldots, n=0,1, \ldots, 2^{k}-1, m \in Z$.

The set of CAS wavelets forms an orthonormal basis of $L^{2}([0,1])$, so any function $f$ which is square integrable in the interval $[0,1]$, it can be expanded as the following form:

$$
f=\sum_{n=0}^{+\infty} \sum_{m \in Z} c_{n, m} \psi_{n, m} \cong \sum_{n=0}^{2^{k}-1} \sum_{m=-M}^{M} c_{n, m} \psi_{n, m} .
$$

Using (1) and (2), the definite integral $\int_{0}^{1} f(x) d x$ is approximated by 


$$
\int_{0}^{1} f(x) d x \cong \int_{0}^{1} \sum_{n=0}^{2^{k}-1} \sum_{m=-M}^{M} c_{n, m} \psi_{n, m}(x) d x=\frac{1}{2^{\frac{k}{2}}} \sum_{n=0}^{2^{k}-1} c_{n, 0} .
$$

To obtain the CAS wavelets coefficients $c_{n, 0}$, we use the collocation points as

$$
x_{j}=\frac{2 j-1}{2^{k+1}(2 M+1)}, j=1, \ldots, 2^{k}(2 M+1) .
$$

Therefore, by substituting these points in (2), we have:

$$
f\left(x_{j}\right)=\sum_{n=0}^{2^{k}-1} \sum_{m=-M}^{M} c_{n, m} \psi_{n, m}\left(x_{j}\right), j=1, \ldots, 2^{k}(2 M+1),
$$

by solving this linear system of equations, we get the coefficients $c_{n, 0}$. Hence, the quadrature rule for single integrals [7] is given by:

$$
\int_{0}^{1} f(x) d x \approx \frac{1}{2^{k}(2 M+1)} \sum_{i=1}^{2^{k}} f\left(\frac{2 i-1}{2^{k+1}(2 M+1)}\right),
$$

and generally for the integral $\int_{a}^{b} f(x) d x$, we have:

$$
\int_{a}^{b} f(x) d x \approx \frac{b-a}{2^{k}(2 M+1)} \sum_{i=1}^{2^{k}} f\left(a+\frac{(b-a)(2 i-1)}{2^{k+1}(2 M+1)}\right) .
$$

Now, by applying the formula (7), we can obtain quadrature rules for double and triple integrals as the following forms:

$$
\int_{a}^{b} \int_{h(y)}^{g(y)} f(x, y) d x d y \approx \frac{b-a}{2^{k}(2 M+1)} \sum_{i=1}^{2^{k}} \sum^{2 M+1)} H\left(a+\frac{(b-a)(2 i-1)}{2^{k+1}(2 M+1)}\right),
$$

where $H(y)=\frac{g(y)-h(y)}{2^{k}(2 M+1)}$.

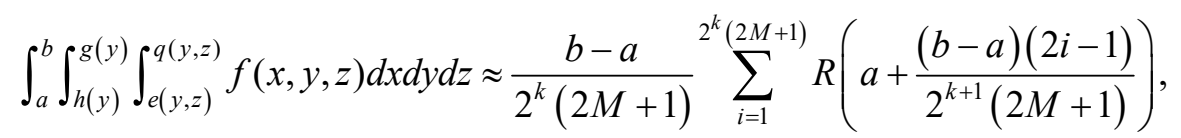

where $\quad R(z)=\left(\frac{g(z)-h(z)}{2^{k}(2 M+1)}\right)^{2^{k}} \sum_{i=1}^{(2 M+1)} H\left(h(z)+\frac{(g(z)-h(z))(2 i-1)}{2^{k+1}(2 M+1)}, z\right) \quad$ and $H(y, z)=\left(\frac{q(y, z)-e(y, z)}{2^{k}(2 M+1)}\right)^{2^{k}} \sum_{i=1}^{2 M+1)} f\left(e(y, z)+\frac{(q(y, z)-e(y, z))(2 i-1)}{2^{k+1}(2 M+1)}, y, z\right)$. 


\subsection{Haar wavelets}

The Haar wavelets basis defined on the interval $[a, b)$ is a family of functions defined on subintervals of $[a, b)$ generated from the scaling and wavelet functions by the dilation and translation [8], these functions are given by:

The scaling function:

$$
h_{1}(x)=\left\{\begin{array}{lc}
1, & \text { for } x \in[a, b) \\
0, & \text { otherwise }
\end{array}\right.
$$

and the wavelet function:

$$
h_{2}(x)=\left\{\begin{array}{lc}
1, & \text { for } x \in\left[a, \frac{a+b}{2}\right) \\
-1, & \text { for } x \in\left[\frac{a+b}{2}, b\right] \\
0, & \text { otherwise. }
\end{array}\right.
$$

Now, for the other functions $h_{i}$ :

$$
h_{i}(x)=\left\{\begin{array}{cc}
1, & \text { for } x \in[\alpha, \beta), \\
-1, & \text { for } x \in[\alpha, \beta), \\
0, & \text { otherwise, }
\end{array}\right.
$$

where

$\alpha=a+(b-a) \frac{k}{m}, \beta=a+(b-a) \frac{k+0.5}{m}, \gamma=a+(b-a) \frac{k+1}{m}, i=3,4, \ldots, 2 M$.

The integer $m=2^{j}$, where $j=0,1, \ldots, J, J=2^{M}, k=0,1, \ldots, m-1, i=m+k+1$.

Thus, any function $f \in L^{2}([a, b))$ can be expressed as

$$
f(x)=\sum_{i=1}^{+\infty} a_{i} h_{i}(x) .
$$

Using the collocation method with Haar wavelets, we obtain the following formula for single integrals:

$$
\int_{a}^{b} f(x) d x \approx \frac{b-a}{2 M} \sum_{k=1}^{2 M} f\left(a+\frac{(b-a)(k-0.5)}{2 M}\right) .
$$

For double and triple integrals, one can refer to $[8,9]$. 


\section{Numerical integration using hybrid functions}

The Hybrid functions family $\psi_{i, j}, i=1,2, \ldots, n, j=0,1, \ldots, m-1$ is defined on the interval $[0,1)$ by:

$$
\psi_{i, j}(x)=\left\{\begin{array}{cc}
L_{j}(2 n x-2 i+1), & \text { for } x \in\left[\frac{i-1}{n}, \frac{i}{n}\right), \\
0, & \text { otherwise }
\end{array}\right.
$$

where, $L_{0}(x)=1, L_{1}(x)=x, L_{k+1}(x)=\left(\frac{2 k+1}{k+1}\right) x L_{k}(x)-\left(\frac{k}{k+1}\right) L_{k-1}(x), k=1,2, \ldots$ So, any function $f \in L^{2}([0,1))$ can be expressed as

$$
f(x)=\sum_{i=1}^{+\infty} \sum_{j=0}^{+\infty} c_{i, j} \psi_{i, j}(x)
$$

Using the collocation method for hybrid functions, we get the integration formula for single integrals with different values of $m$ as in [8]:

For $m=1$,

$$
\int_{0}^{1} f(x) d x \approx \frac{1}{n} \sum_{i=1}^{n} f\left(\frac{2 i-1}{2 n}\right)
$$

For $m=2$,

$$
\int_{0}^{1} f(x) d x \approx \frac{1}{2 n} \sum_{i=1}^{2 n} f\left(\frac{2 i-1}{4 n}\right)
$$

For $m=3$,

$$
\int_{0}^{1} f(x) d x \approx \frac{1}{8 n} \sum_{i=1}^{n}\left[3 f\left(\frac{6 i-5}{6 n}\right)+2 f\left(\frac{6 i-3}{6 n}\right)+3 f\left(\frac{6 i-1}{6 n}\right)\right] .
$$

For approximate values of double and triple integrals with different values of $m$, one can refer to $[8,9]$.

\section{Proposed numerical integration method}

The aim of this section is to develop a new numerical integration method that overcomes the previous three methods for single, double and triple integrals in terms of absolute errors. 


\subsection{Coifman wavelets overview}

In [11], an orthonormal wavelet basis is called a Coifman wavelet basis (Coiflet) of degree $N, N=1,2, \ldots$, if the corresponding scaling function $\varphi$ and wavelet $\psi$ satisfy:

$$
\begin{gathered}
\operatorname{supp} \varphi=\operatorname{supp} \psi \subset[0,6 N-1] \\
\int_{-\infty}^{+\infty} \varphi(x) d x=1, \int_{-\infty}^{+\infty} x^{\ell} \varphi(x) d x=0, \ell=1,2, \ldots, 2 N-1, \\
\int_{-\infty}^{+\infty} x^{\ell} \psi(x) d x=0, \ell=0,1, \ldots, 2 N-1 .
\end{gathered}
$$

The Coifman scaling function $\varphi$ of degree $N$ verifies the following properties that will be useful in our study:

$$
\begin{gathered}
\sum_{k=-\infty}^{+\infty} \varphi(x-k)=1, \forall x \in(-\infty,+\infty), \\
\sum_{k=-\infty}^{+\infty}(x-k)^{\ell} \varphi(x-k)=0, \ell=1,2, \ldots, 2 N-1, \forall x \in(-\infty,+\infty) .
\end{gathered}
$$

More details and other properties of coiflets, one can refer to [12].

\subsection{Numerical formula for single integrals using coiflets}

Definition 4.1. Let $\varphi$ be the Coifman scaling function of degree $N$ and $f$ be a function defined on $[1-6 N, 6 N-1]$. The coiflet sampling approximation of $f$ at level $j,(j \geq 0)$ on the interval $[0,6 N-1]$ is defined by:

$$
S_{j} f(x)=2^{-\frac{j}{2}} \sum_{k=1-6 N}^{(6 N-1) 2^{j}} f\left(\frac{k}{2^{j}}\right) \varphi_{j, k}(x), x \in[0,6 N-1],
$$

where $\varphi_{j, k}(x)=2^{\frac{j}{2}} \varphi\left(2^{j} x-k\right)$.

The following theorem provides the numerical formula for single integrals.

Theorem 4.2. Let $a<b$ and $f \in C^{2 N}([2 a-b, b])$. For a Coifman scaling function $\varphi$ of degree $N$ and $j \geq 0$, we define $I_{j} f$ by:

$$
I_{j} f=\frac{(b-a)}{(6 N-1) 2^{j}} \sum_{k=2-6 N}^{(6 N-1) 2^{j}-1}\left(\int_{-k}^{-k+(6 N-1) 2^{j}} \varphi(x) d x\right) f\left(a+\left(\frac{b-a}{6 N-1}\right) \frac{k}{2^{j}}\right),
$$


then $I_{j} f$ is an approximate value of the integral $\int_{a}^{b} f(x) d x$ whose the error estimation is evaluated by:

$$
\left|\int_{a}^{b} f(x) d x-I_{j} f\right| \leq C 2^{-2 N j}
$$

where $C$ depends only on $f$ and $\varphi$.

To demonstrate the previous theorem, we need to provide the following lemmas.

Lemma 4.3. Let $\varphi$ be the Coifman scaling function of degree $N$, then we have:

$$
\sum_{k=1-6 N}^{(6 N-1) 2^{j}} \varphi\left(2^{j} x-k\right)=1, x \in[0,6 N-1]
$$

and

$$
\sum_{k=1-6 N}^{(6 N-1) 2^{j}}\left(2^{j} x-k\right)^{\ell} \varphi\left(2^{j} x-k\right)=0, \ell=1,2, \ldots, 2 N-1, x \in[0,6 N-1]
$$

Lemma 4.4. Let us consider $f \in C^{2 N}([1-6 N, 6 N-1])$ and $S_{j} f$ be its coiflet sampling approximation on $[0,6 N-1]$, then we have:

$$
\left\|f-S_{j} f\right\|_{L^{2}([0,6 N-1])} \leq C 2^{-2 N j}
$$

where $C$ depends only on $f$ and $\varphi$.

Proof of Lemma 4.3. The proof is easily derived, when we use the relations (20), (23) and (24).

Proof of Lemma 4.4. For $k=1-6 N, \ldots,(6 N-1) 2^{j}$ and $0 \leq x \leq 6 N-1$, the Taylor expansion of $f$ at the point $x$ gives:

$$
f\left(\frac{k}{2^{j}}\right)=f(x)+\sum_{\ell=1}^{2 N-1} \frac{f^{(\ell)}(x)}{\ell !}\left(\frac{k}{2^{j}}-x\right)^{\ell}+\frac{f^{(2 N)}\left(\alpha_{k}\right)}{(2 N) !}\left(\frac{k}{2^{j}}-x\right)^{2 N} .
$$

We multiply by $2^{-\frac{j}{2}} \varphi_{j, k}(x)$ and we sum the result, we obtain

$$
\begin{aligned}
S_{j} f(x) & =f(x) 2^{-\frac{j}{2}} \sum_{k=1-6 N}^{(6 N-1) 2^{j}} \phi_{j, k}(x)+2{ }^{-\frac{j}{2}} \sum_{\ell=1}^{2 N-1} \frac{f^{(\ell)}(x)}{\ell !} \sum_{k=1-6 N}^{(6 N-1) 2^{j}}\left(\frac{k}{2^{j}}-x\right)^{\ell} \varphi_{j, k}(x) \\
& +2^{-\frac{j}{2}} \sum_{k=1-6 N}^{(6 N-1) 2^{j}} \frac{f^{(2 N)}\left(\alpha_{k}\right)}{(2 N) !}\left(\frac{k}{2^{j}}-x\right)^{2 N} \varphi_{j, k}(x)
\end{aligned}
$$


Using (28) and (29) of the Lemma 4.3, we get

$$
S_{j} f(x)=f(x)+\frac{1}{(2 N) ! 2^{2 N j}} \sum_{k=1-6 N}^{(6 N-1) 2^{j}} f^{(2 N)}\left(\alpha_{k}\right)\left(2^{j} x-k\right)^{2 N} \varphi\left(2^{j} x-k\right) .
$$

Then,

$\left\|f-S_{j} f\right\|_{L^{2}([0,6 N-1])}^{2}=\frac{1}{\left((2 N) ! 2^{2 N j}\right)^{2}}\left\|\sum_{k=1-6 N}^{(6 N-1) 2^{j}} f^{(2 N)}\left(\alpha_{k}\right)\left(2^{j} x-k\right)^{2 N} \varphi\left(2^{j} x-k\right)\right\|_{L^{2}([0,6 N-1])}^{2}$

If we put, $g_{k}(x)=\left(2^{j} x-k\right)^{2 N} \varphi\left(2^{j} x-k\right)$, then $\operatorname{supp}\left(g_{k}\right) \subset\left[\frac{k}{2^{j}}, \frac{k+6 N-1}{2^{j}}\right]$ and by using the regularity of $\varphi$, we have $\exists C_{N}>0,\left|g_{k}(x)\right| \leq C_{N}, \forall x \in(-\infty,+\infty)$. Also we assume that $M=\sup _{|x| 66 N-1}\left|f^{(2 N)}(x)\right|$, then

$$
\begin{aligned}
\left\|\sum_{k=1-6 N}^{(6 N-1) 2^{j}} f^{(2 N)}\left(\alpha_{k}\right)\left(2^{j} x-k\right)^{2 N} \varphi\left(2^{j} x-k\right)\right\|_{L^{2}([0,6 N-1])}^{2} \\
\leq M^{2} \sum_{k=1-6 N}^{(6 N-1) 2^{j}} \sum_{k^{\prime}=1-6 N+k}^{6 N-1+k} \int_{\frac{k}{2^{j}}}^{\frac{k+6 N-1}{2^{j}}}\left|g_{k}(x) g_{k^{\prime}}(x)\right| d x \\
\leq 9 M^{2} C_{N}{ }^{2}(6 N-1)^{3}
\end{aligned}
$$

Therefore, $\left\|f-S_{j} f\right\|_{L^{2}([0,6 N-1])} \leq \frac{3 M C_{N}(6 N-1)^{\frac{3}{2}}}{(2 N) !} 2^{-2 N j}$.

Proof of Theorem 4.2. By a change of variable $t=(6 N-1) \frac{x-a}{b-a}$, we find that

$$
\int_{a}^{b} f(x) d x=\int_{0}^{6 N-1}\left(\frac{b-a}{6 N-1}\right) f\left(a+\left(\frac{b-a}{6 N-1}\right) t\right) d t
$$

then the function $g$ defined by $g(t)=\left(\frac{b-a}{6 N-1}\right) f\left(a+\left(\frac{b-a}{6 N-1}\right) t\right)$ belongs to $C^{2 N}([1-6 N, 6 N-1])$. 
By applying the Lemma 4.4, we obtain

$$
\begin{aligned}
\left|\int_{0}^{6 N-1} g(t) d t-\int_{0}^{6 N-1} S_{j} g(t) d t\right| \leq \int_{0}^{6 N-1} \mid g(t)- & S_{j} g(t) \mid d t \\
& \leq \sqrt{\int_{0}^{6 N-1} d t} \sqrt{\int_{0}^{6 N-1}\left|g(t)-S_{j} g(t)\right|^{2} d t} \\
& \leq \frac{3 M C_{N}(6 N-1)^{2}}{(2 N) !} 2^{-2 N j} .
\end{aligned}
$$

On the other hand, we have

$$
\int_{0}^{6 N-1} S_{j} g(x) d x=2^{-j} \sum_{k=1-6 N}^{(6 N-1) 2^{j}} g\left(\frac{k}{2^{j}}\right)^{-k+(6 N-1) 2^{j}} \int_{-k} \varphi(x) d x .
$$

Since $\int_{0}^{6 N-1} g(t) d t=\int_{a}^{b} f(x) d x$ and $\int_{0}^{6 N-1} S_{j} g(t) d t=I_{j} f$, then the proof is complete.

Remark 4.5. Since the Coifman scaling function does not have a closed form, its integrals are determined iteratively by the cascade algorithm with good approximation.

\subsection{Numerical formula for double integrals using coiflets}

Consider the following integral:

$$
\int_{a}^{b} \int_{c(y)}^{d(y)} F(x, y) d x d y
$$

Applying the formula (26) to the integral $\int_{c(y)}^{d(y)} F(x, y) d x$, we get

$\int_{c(y)}^{d(y)} F(x, y) d x \approx \frac{(d(y)-c(y))}{(6 N-1) 2^{j_{1}}} \sum_{k=2-6 N}^{(6 N-1) 2^{j_{1}}}-1\left(\int_{-k}^{-k+(6 N-1) 2^{j_{1}}} \varphi(x) d x\right) F\left(c(y)+\left(\frac{d(y)-c(y)}{6 N-1}\right) \frac{k}{2^{j_{1}}}, y\right)$

Now, we put

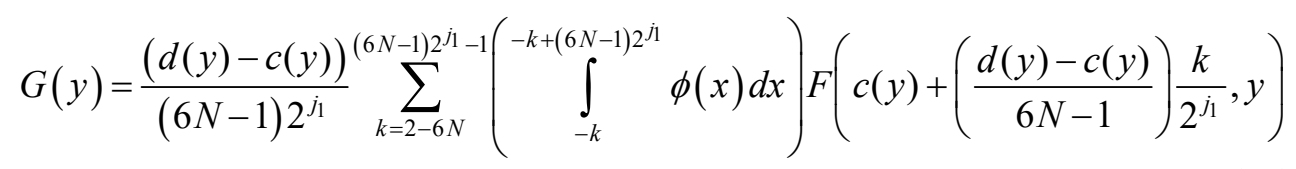

We apply the formula (26) once again, we obtain the numerical formula of double integrals with variable limits as: 


$$
\begin{gathered}
\int_{a}^{b} \int_{c(y)}^{d(y)} F(x, y) d x d y \approx \int_{a}^{b} G(y) d y \approx \\
\frac{(b-a)}{(6 N-1) 2^{j_{2}}} \sum_{k=2-6 N}^{(6 N-1) 2^{j_{2}}-1}\left(\int_{-k}^{-k+(6 N-1) 2^{j_{2}}} \varphi(x) d x\right) G\left(a+\left(\frac{b-a}{6 N-1}\right) \frac{k}{2^{j_{2}}}\right)
\end{gathered}
$$

Note that we may take $j_{1}=j_{2}$.

\subsection{Numerical formula for triple integrals using coiflets}

The numerical formula for triple integrals is obtained in a similar way and is given by:

$$
\begin{gathered}
\int_{a}^{b} \int_{c(z)}^{d(z)} \int_{e(y, z)}^{f(y, z)} F(x, y, z) d x d y d z \approx \\
\frac{(b-a)}{(6 N-1) 2^{j_{3}}} \sum_{k=2-6 N}^{(6 N-1) 2^{j_{3}}-1}\left(\int_{-k}^{-k+(6 N-1) 2^{j_{3}}} \varphi(x) d x\right) H\left(a+\left(\frac{b-a}{6 N-1}\right) \frac{k}{2^{j_{3}}}\right)
\end{gathered}
$$

where

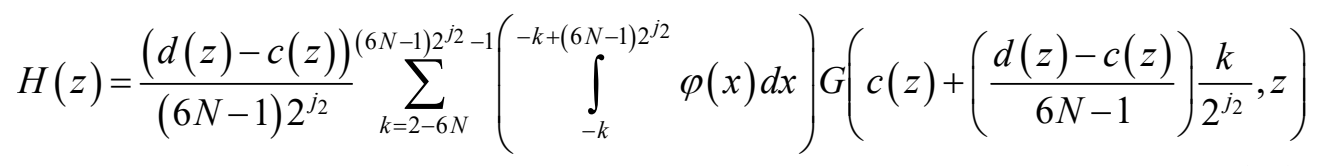

and

$$
\begin{gathered}
G(y, z)=\frac{(f(y, z)-e(y, z))}{(6 N-1) 2^{j_{1}}} \sum_{k=2-6 N}^{(6 N-1) 2^{j_{1}}-1}\left(\int_{-k}^{-k+(6 N-1) 2^{j_{1}}} \varphi(x) d x\right) F(e(y, z)+ \\
\left.\left(\frac{f(y, z)-e(y, z)}{6 N-1}\right) \frac{k}{2^{j_{1}}}, y, z\right)
\end{gathered}
$$

\section{Numerical examples}

In this section we give numerical experiments to illustrate the efficiency of our proposed method. Using our approach, the algorithms have been implemented in Matlab using coiflets of degree 1 and 2 with 7 iterations. So, the numerical results are compared with exact solutions as presented in tables. 
Example 5.1. Consider the following integral:

$$
\int_{0}^{1} \frac{1}{1+x^{2}} d x=\frac{\pi}{4}
$$

Absolute errors of four methods' applied to numerical calculation of the single integral are shown in Table 1.

Table 1. Comparison of absolute errors for single integral

\begin{tabular}{|c|c|c|}
\hline Methods & Parameters & Absolute Errors \\
\hline \multirow{3}{*}{ CAS Wavelets } & $r=5, k=1$ & $2.08333 \mathrm{e}-004$ \\
\cline { 2 - 3 } & $r=11, k=1$ & $4.30441 \mathrm{e}-005$ \\
\cline { 2 - 3 } & $r=13, k=4$ & $4.81540 \mathrm{e}-007$ \\
\hline \multirow{3}{*}{ Haar Wavelets } & $M=4$ & $3.25519 \mathrm{e}-004$ \\
\cline { 2 - 3 } & $M=8$ & $8.13802 \mathrm{e}-005$ \\
\cline { 2 - 3 } Hybrid Functions & $M=16$ & $2.03451 \mathrm{e}-005$ \\
\hline \multirow{3}{*}{ Proposed Method } & $m=1, n=10$ & $2.08333 \mathrm{e}-004$ \\
\cline { 2 - 3 } & $m=2, n=15$ & $2.31481 \mathrm{e}-005$ \\
\cline { 2 - 3 } & $m=2, n=20$ & $1.30208 \mathrm{e}-005$ \\
\cline { 2 - 3 } & $N=1, j=10$ & $1.66929 \mathrm{e}-004$ \\
\hline \multirow{3}{*}{} & $N=1, j=15$ & $5.21712 \mathrm{e}-006$ \\
\hline
\end{tabular}

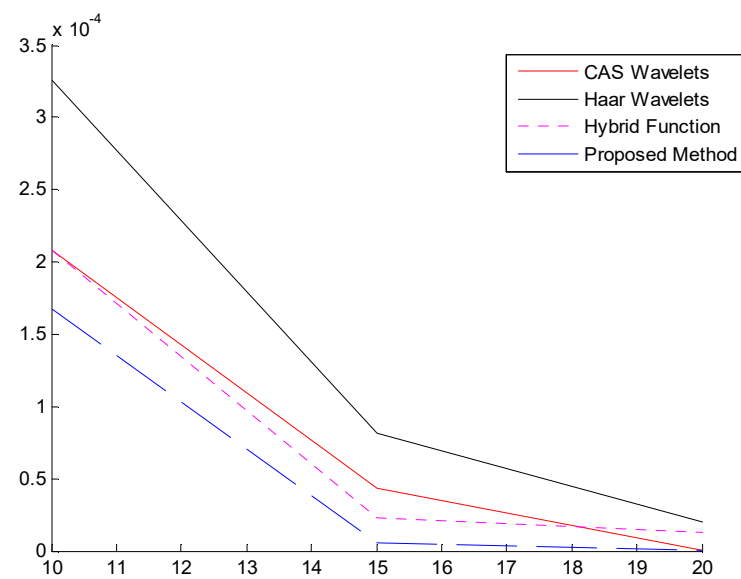

Fig. 1. Error graphs of Example 5.1 using four methods at different parameters

Example 5.2. Consider the following integral:

$$
\int_{0}^{1} \int_{0}^{\sqrt{1+y^{2}}} \frac{1}{1+x^{2}+y^{2}} d x d y=-\frac{\pi}{4} \log (\sqrt{2}-1) .
$$


Absolute errors of four methods' applied to numerical calculation of the double integral are shown in Table 2.

Table 2. Comparison of absolute errors for double integral

\begin{tabular}{|c|c|c|}
\hline Methods & Parameters & Absolute Errors \\
\hline \multirow{3}{*}{ CAS Wavelets } & $r=3, k=1$ & $8.31863 \mathrm{e}-004$ \\
\cline { 2 - 3 } & $r=7, k=2$ & $3.81794 \mathrm{e}-005$ \\
\cline { 2 - 3 } & $r=11, k=3$ & $3.86519 \mathrm{e}-006$ \\
\hline \multirow{3}{*}{ Haar Wavelets } & $M=4$ & $4.67821 \mathrm{e}-004$ \\
\cline { 2 - 3 } & $M=8$ & $1.16930 \mathrm{e}-004$ \\
\cline { 2 - 3 } & $M=16$ & $2.92310 \mathrm{e}-005$ \\
\hline \multirow{3}{*}{ Hybrid Functions } & $m=1, n=10$ & $2.99375 \mathrm{e}-004$ \\
\cline { 2 - 3 } & $m=2, n=15$ & $3.32584 \mathrm{e}-005$ \\
\cline { 2 - 3 } & $m=2, n=20$ & $1.87077 \mathrm{e}-005$ \\
\hline \multirow{3}{*}{ Proposed Method } & $N=1, j=10$ & $2.00733 \mathrm{e}-004$ \\
\cline { 2 - 3 } & $N=1, j=15$ & $2.40027 \mathrm{e}-006$ \\
\cline { 2 - 3 } & $N=1, j=20$ & $7.50087 \mathrm{e}-008$ \\
\hline
\end{tabular}

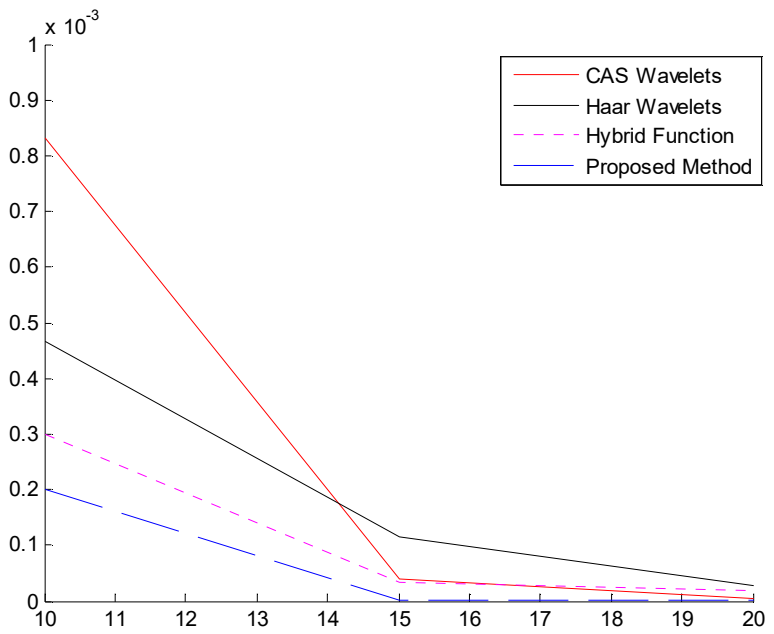

Fig. 2. Error graphs of Example 5.2 using four methods at different parameters

Example 5.3. Consider the following integral:

$$
\int_{0}^{\pi} \int_{0}^{z} \int_{0}^{z y} \frac{1}{y} \sin \left(\frac{x}{y}\right) d x d y d z=\frac{\left(4+\pi^{2}\right)}{2} .
$$


Absolute errors of four methods' applied to numerical calculation of the triple integral are shown in Table 3.

Table 3. Comparison of absolute errors for triple integral

\begin{tabular}{|c|c|c|}
\hline \multicolumn{1}{|c|}{ Methods } & Parameters & Absolute Errors \\
\hline \multirow{4}{*}{ CAS Wavelets reported in [7] } & $r=3, k=1$ & $2.46417 \mathrm{e}-002$ \\
\cline { 2 - 3 } & $r=7, k=4$ & $7.38043 \mathrm{e}-005$ \\
\cline { 2 - 3 } & $r=9, k=4$ & $9.97127 \mathrm{e}-005$ \\
\hline \multirow{3}{*}{ Haar Wavelets reported in [9] } & $M=8$ & $3.5959 \mathrm{e}-003$ \\
\cline { 2 - 3 } & $M=16$ & $9.0291 \mathrm{e}-004$ \\
\cline { 2 - 3 } & $M=32$ & $2.2597 \mathrm{e}-004$ \\
\hline \multirow{3}{*}{ Hybrid Functions reported in [9] } & $m=3, n=20$ & $2.4465 \mathrm{e}-007$ \\
\cline { 2 - 3 } & $m=4, n=20$ & $1.2654 \mathrm{e}-007$ \\
\cline { 2 - 3 } & $m=5, n=20$ & $4.2473 \mathrm{e}-0011$ \\
\hline \multirow{3}{*}{ Proposed Method } & $N=1, j=20$ & $2.21819 \mathrm{e}-007$ \\
\cline { 2 - 3 } & $N=2, j=15$ & $1.02981 \mathrm{e}-008$ \\
\cline { 2 - 3 } & $N=2, j=20$ & $6.43639 \mathrm{e}-0013$ \\
\hline
\end{tabular}

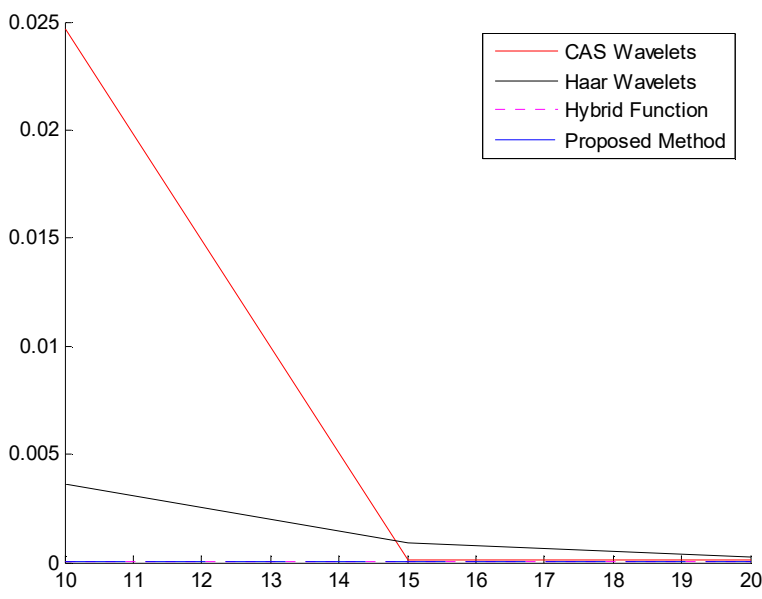

Fig. 3. Error graphs of Example 5.3 using four methods at different parameters

Obviously, the numerical results and error graphs (Figs. 1-3) about these examples show that the absolute errors of our proposed method using different levels are smaller and decrease more quickly than those obtained by the three different methods. Moreover, due to the mathematical properties of coiflets, the differences in the obtained errors between our proposed method and other methods are very significant. Then, this leads to a faster and more accurate convergence for our method. 


\section{Conclusions}

In this paper, a new numerical integration method based on coiflets sampling approximation has been applied for single, double and triple integrals with variable limits. The comparison between four methods shows that our proposed method gives better results than CAS wavelets, Haar wavelets and Hybrid functions in terms of absolute errors.

\section{References}

[1] François, D. (2016). Revisited optimal error bounds for interpolatory integration rules. Advances in Numerical Analysis, Vol. 2016, 1-8, DOI: 10.1155/2016/3170595.

[2] Sharifi, M.A., \& Seif, M.R. (2014). A new family of multistep numerical integration methods based on Hermite interpolation. Celestial Mechanics and Dynamical Astronomy, 118(1), 29-48, DOI: $10.1007 / \mathrm{s} 10569-013-9517-4$.

[3] Zlatko, U. (2006). Some modifications of the trapezoidal rule. Sarajevo Journal of Mathematics, 2(2), 237-245, http://www.anubih.ba/Journals/vol-2,no-2,y06/13revudovicic.pdf

[4] Dehda, B., \& Melkemi, K. (2017). Image denoising using new wavelet thresholding function. Journal of Applied Mathematics and Computational Mechanics, 16(2), 55-65, DOI: 10.17512/ jamcm.2017.2.05.

[5] Dehda, B., \& Melkemi, K. (2016). Novel method for reduction of wavelet coefficients number and its applications in images compression. International Journal of Applied Mathematics and Machine Learning, 5(1), 43-65, DOI: 10.18642/ijamml_7100121693.

[6] Imran, A. (2013). New algorithms for the numerical solution of nonlinear Fredholm and Volterra integral equations using Haar wavelets. Journal of Computational and Applied Mathematics, 239, 333-345, DOI: 10.1016/j.cam.2012.08.031.

[7] Rezabeyk, S., \& Maleknejad, K. (2015). Application of CAS wavelet to construct quadrature rules for numerical integration. Int. J. Industrial Mathematics, 7(1), 87-92, http://ijim.srbiau.ac.ir/

[8] Imran, A., \& Fazal, H. (2010). A comparative study of numerical integration based on Haar wavelets and hybrid functions. Computers and Mathematics with Applications, 59(6), 2026-2036, DOI: 10.1016/j.camwa.2009.12.005.

[9] Imran, A., \& Wajid, K. (2011). Quadrature rules for numerical integration based on Haar wavelets and hybrid functions. Computers and Mathematics with Applications, 61, 2770-2781, DOI: 10.1016/j.camwa.2011.03.043.

[10] Barzkar, A., \& Assari, P. (2012). Application of the CAS Wavelet in solving Fredholm-Hammerstein integral equations of the second kind with error analysis. World Applied Sciences Journal, 18(12), 1695-1704, DOI: 10.5829/idosi.wasj.2012.18.12.467.

[11] Černá, D., Finěk, V., \& Najzar, K. (2008). On the exact values of coefficients of coiflets. Cent. Eur. J. Math., 6(1), 159-169, DOI: 10.2478/s11533-008-0011-2.

[12] Xiaomin, W. (2014). A Coiflets-based wavelet Laplace method for solving the Riccati differential equations. Journal of Applied Mathematics, Vol. 2014, 1-8, DOI: 10.1155/2014/257049. 